\title{
Fault-tolerant Distribution Network Enabled by Series Soft Open Point
}

This paper was downloaded from TechRxiv (https://www.techrxiv.org).

LICENSE

CC BY 4.0

SUBMISSION DATE / POSTED DATE

$15-06-2021$ / 22-06-2021

CITATION

Qi, Yang (2021): Fault-tolerant Distribution Network Enabled by Series Soft Open Point. TechRxiv. Preprint. https://doi.org/10.36227/techrxiv.14782368.v1

$\mathrm{DOI}$

10.36227/techrxiv.14782368.v1 


\section{Fault-tolerant Distribution Network Enabled by Series Soft Open Point}

\author{
Yang Qi \\ Department of Electrical Engieering \\ Northwestern Polytechnical University \\ Xi'an City, Shaanxi Province, China \\ qiyang@nwpu.edu.cn
}

\author{
Han Deng \\ Interdisciplanry Graduate Program \\ Nanyang Technological Univeristy \\ Singapore \\ han017@e.ntu.edu.sg
}

\author{
Yi Tang \\ School of Electrical and Electronic \\ Engineering \\ Nanyang Technological Univeristy \\ Singapore \\ yitang@ntu.edu.sg
}

\begin{abstract}
Reliability enhancement is always considered as the top priority of the power distribution network. It is highly expected that the power network faults can be locally addressed without spreading into the entire grid. To fulfill the requirement, this paper develops a smart power-electronics device named series soft open point (SSOP). The proposed SSOP connects two AC terminals and can suppress the surge current caused by a short-circuit fault. In addition, it also performs the grid-forming function during an islanding fault. The principle of SSOP will be elaborated and the effectiveness of this proposal is validated by experimental results.
\end{abstract}

Keywords-Distribution network, Fault-tolerant control, Softopen point.

\section{INTRODUCTION}

Recent advances in power electronics technologies have greatly changed the characteristics of distribution network [1]. With more and more distributed generations (DGs) being integrated through power converters [2]-[4], the fault-tolerant operation of power grid becomes an emerging and important aspect for the future distribution network. It is therefore of great importance to locate faults and minimize their adverse impacts. To realize this target, a number of fault-diagnosis and fault-tolerant operation schemes have been proposed in the existing literature [5]-[8].

Among them, one common pursuit is to confine the fault within the local area so that the adverse impact on the rest of system can be minimized. Generally, the fulfillment of this target depends on the network topology. For the distribution network with a radial configuration, there are no redundant links between adjacent feeders. Therefore, the power supply of some branches will be affected when the islanding faults happen and isolate these branches from the rest of networks. In comparison, the distribution network with a networked topology is more robust against islanding faults due to massive interconnections. However, this attribute will also bring other challenges, as the short-circuit faults can easily spread into the rest of distribution network [9].

To merge the merits of radial and networked power grids, power-electronic-interfaced soft open point (SOPs) have been developed, which can prevent the short-circuit current from spreading out and provide an alternative route of electricity supply during the islanded faults [10]-[11]. The existing SOPs usually has a back-to-back configuration with two or multiple converters sharing the same DC link. One converter (master unit) is responsible for regulating the DC-link voltage while the other (slave units) regulate the active and reactive power flow [12]-[13]. However, the system reliability will be compromised by this master-slave configuration. To be specific, malfunctions will happen if the short-circuit or islanding fault occurs at the $\mathrm{AC}$ port of the master converter. Consequently, the master converter cannot maintain the synchronization with the AC power grid. The DC-link voltage cannot be regulated and the entire system would eventually collapse. In addition to this, the slave converter control mode should be switched from the current control mode (CCM) to the voltage control mode (VCM) after an islanding fault happens. The transition would require an extra anti-islanding detection unit and may cause transient voltage dips or sags.

On the other hand, the series-connected power converter, which does not have a back-to-back configuration, provide an alternative solution to the problems. These converters have been deployed to mitigate voltage sag or swell as the dynamic voltage restorer (DVC) [14], regulate the voltage magnitude as the dynamic voltage conditioner (DVR) [15], and manage the load consumption as the electric spring [16]-[17]. In comparison with the parallel-connected converters, the series converters can directly make a voltage compensation between the two AC terminals.

In view of the above discussions, this paper develops an series soft open point (SSOP) and its control strategy. The proposed solution can autonomously handle short-circuit and islanding faults that occur at any of the two AC terminals. In addition to this, the number of power semiconductors can be reduced by half, as compared with the same (half bridge or full bridge) back-to-back configuration. The principle behind will be elaborated and hardware experiments are conducted for verification.

\section{Circuit CONFIGURATION}

Fig. 1 illustrates the system circuit configuration, where the SOP is connected in series between the two AC terminals of the distribution network. Each AC terminal contains an equivalent AC voltage source $v_{g}$ and the aggregated load $R_{l}$. Besides, $R_{g}$ and $L_{g}$ represent line impedance and inductance, respectively. $L_{f}$ and $C_{f}$ are the filter inductance and capacitance. $C_{d c}$ denotes the DC-link capacitor.

Three different operating scenarios for the SSOP have been illustrated. In Fig. 1(a), both AC terminals are in normal conditions and the SSOP creates a current path between the two terminals. Fig. 1(b) shows the scenario of an islanding fault, where $v_{g b}$ is disconnected from terminal $b$ and cannot supply $R_{l b}$. In this case, the SSOP should perform the gridforming function for terminal $b$ even without $v_{g b}$. In Fig. 1(c), a short-circuit fault occurs as terminal $b$ is grounded through a small impedance $Z_{c}$. It should be mentioned that $Z_{c}$ is small enough to drop the voltage magnitude of $v_{b}$. As a result, there exists a considerable voltage difference between the two terminals. The voltage difference, if directly imposed on line impedances, can result in a significant short-circuit current. To prevent this from happening, the SSOP is expected to provide a blocking effect in the short-circuit path, which helps to limit the short-circuit current.

According to Kirchhoff voltage law, the current flow during normal operation equals 


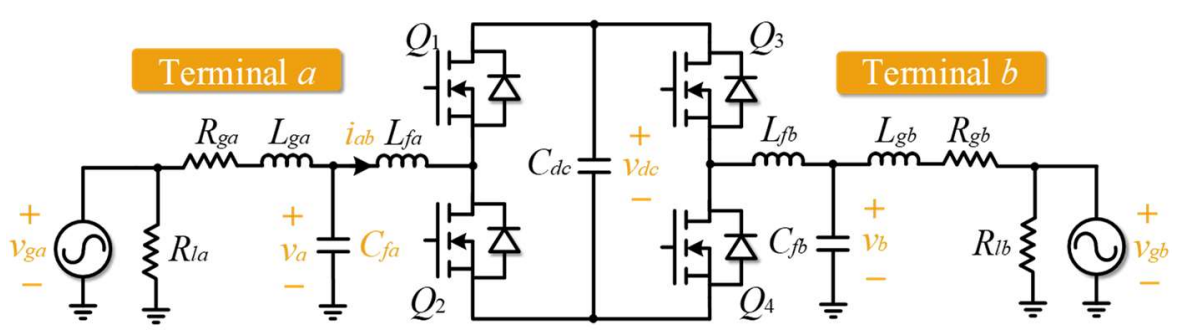

(a)

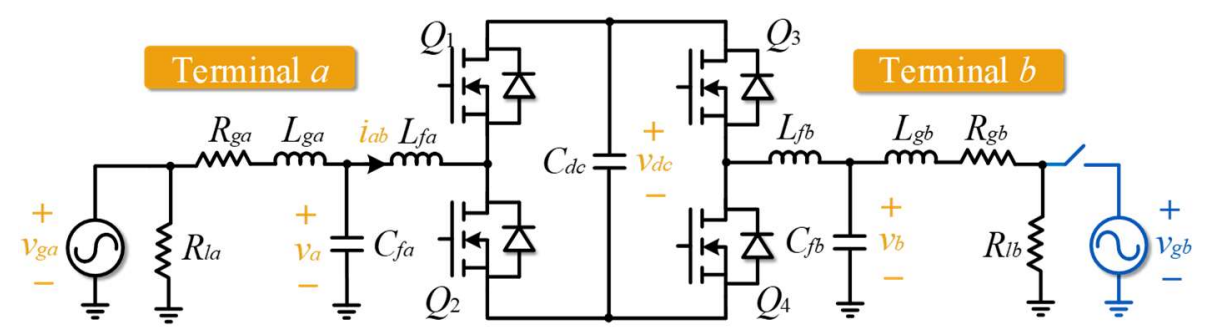

(b)

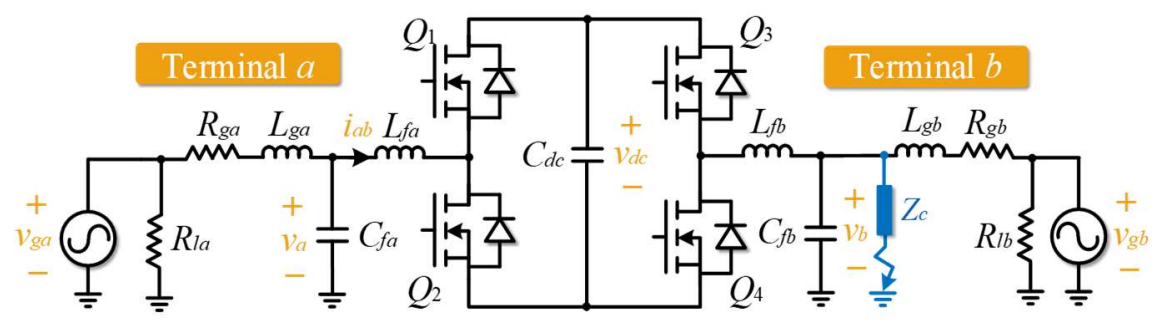

(c)

Fig. 1. Circuit configuration of SSOP under various operating conditions. (a). Normal scenario. (b). Islanding fault. (c). Short-circuit fault.

$$
\begin{aligned}
i_{a b_{-} n}= & \frac{v_{g a}+\left(v_{b}-v_{a}\right)-v_{g b}}{R_{g a}+R_{g b}+j \omega L_{g a}+j \omega L_{g b}} \\
= & \frac{v_{g a}+v_{s}-v_{g b}}{R_{g \Sigma}+j \omega L_{g \Sigma}} .
\end{aligned}
$$

where $R_{g \Sigma}$ is the sum of resistance $R_{g a}$ and $R_{g b} ; L_{g \Sigma}$ is the sum of inductance $L_{g a}$ and $L_{g b} ; v_{s}$ is SSOP output voltage. For the islanding and short-circuit fault, the current flow $i_{a b-o}$ and $i_{a b-s}$ can be respectively expressed as

$$
\begin{gathered}
i_{a b_{-} o}=\frac{v_{g a}+v_{s}}{R_{g a}+R_{l b}+j \omega L_{g a}} . \\
i_{a b_{-} s}=\frac{v_{g a}+v_{s}-v_{b}}{R_{g a}+j \omega L_{g a}} .
\end{gathered}
$$

where $i_{a b_{-} o}$ and $i_{a b_{s} s}$ are current flows under the islanding fault and short-circuit fault, respectively. From the above equations, Fig. 2 shows the typical phasor diagrams for different cases, where $v_{l}$ is the voltage drop of line impedance. For the normal and islanding cases, the difference between $v_{g a}$ and $v_{g b}$ (or $v_{b}$ ) is comparatively small. However, a short-circuit fault usually makes the voltage $v_{b}$ much smaller than $v_{g a}$. This will lead to a large $v_{l}$ and consequently a significant $i_{a b_{-} s}$. To solve this problem, the SSOP can counteract the voltage sag through regulating $v_{s}$, as shown in Fig. 2.
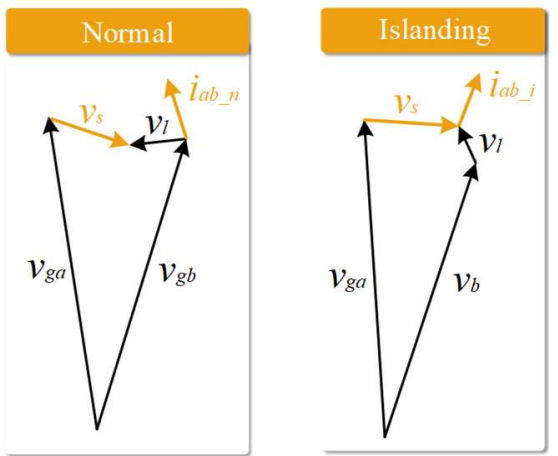

Short circuit

Fig. 2. Typical phasor diagrams under different operating scenarios.

\section{SSOP CONTROL STRATEGY}

Denoting the phase angle between $v_{s}$ and $i_{a b}$ as $\varphi$, the SSOP output voltage $v_{s}$ can be expressed as the sum of quadrature components,

$$
v_{s}=V_{s \alpha}+j V_{s \beta}=V_{s} \cos \varphi+j V_{s} \sin \varphi .
$$

where $V_{s \alpha}$ is in phase with $i_{a b}$ and $j V_{s \beta}$ is orthogonal with $i_{a b}$. The phases of $V_{s \alpha}$ and $j V_{s \beta}$ can be generated by an orthogonalsignal generator (OSG) block. The second order generalized integrator, as one of the most common OSGs, is implemented to achieve this objective. The transfer functions of SOGI are given by 


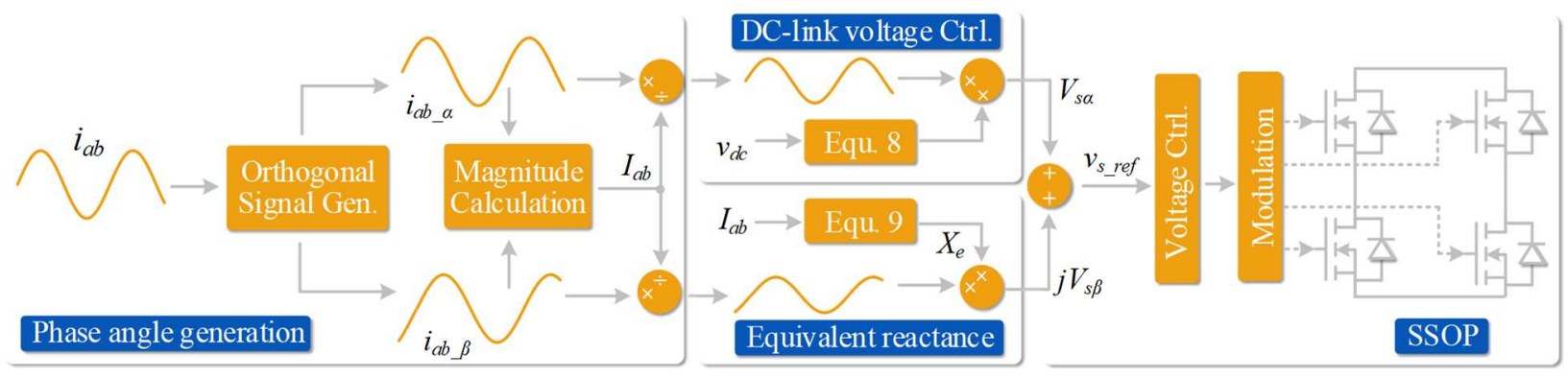

Fig. 3. Control block diagram of the series soft open point.

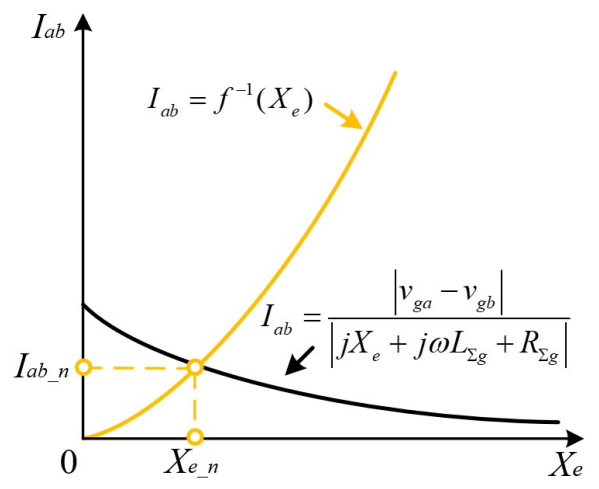

(a)

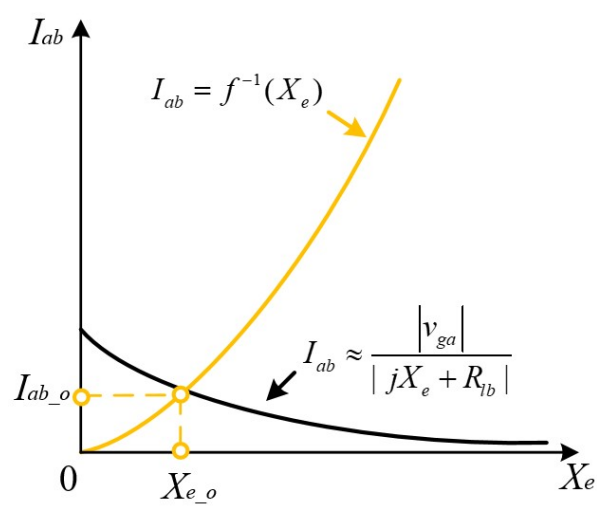

(b)

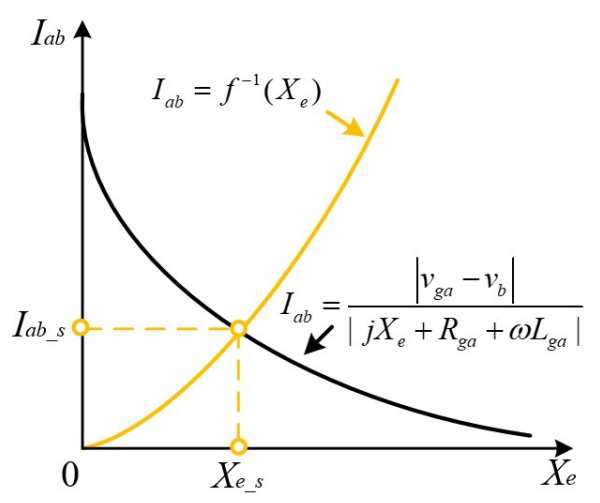

(c)

Fig. 4. Graphical illustration of different scenarios. (a). Normal operation. (b). Islanding fault. (c). Short-circuit fault.

$$
\begin{aligned}
& \frac{i_{a b_{-} \alpha}(s)}{i_{a b}(s)}=\frac{k_{\mathrm{SOGI}} \omega_{n} s}{s^{2}+k_{\mathrm{SOGI}} \omega_{n} s+\omega_{n}^{2}}, \\
& \frac{i_{a b_{-} \beta}(s)}{i_{a b}(s)}=\frac{k_{\mathrm{SOGI}} \omega_{n}^{2}}{s^{2}+k_{\mathrm{SOGI}} \omega_{n} s+\omega_{n}^{2}},
\end{aligned}
$$

where $\omega_{n}$ is the nominal frequency, $k_{\mathrm{SOGI}}$ is a proportional gain, $i_{a b_{-} \alpha}$ is the in-phase component and $i_{a b \beta}$ is the quadrature component. The magnitude of $i_{a b}$ can be calculated by

$$
I_{a b}=\sqrt{i_{a b_{-} \alpha}^{2}+i_{a b_{-} \beta}^{2}} .
$$

The ratio of $i_{a b \_\alpha}$ over $I_{a b}$ provides the angle information for $V_{s \alpha}$, while the ratio of $i_{a b} \beta$ over $I_{a b}$ gives the angle information for $j V_{s \beta}$.

In the steady state, $v_{s}$ is orthogonal with $i_{a b}$ so that no active power is continuously charged or discharged to the DC-link capacitor. To realize this target, a proportional-integral (PI) controller is implemented to regulate the DC-link voltage.

$$
V_{s \alpha}=k_{p}\left(v_{d c}-v_{d c_{-} r e f}\right)+k_{i} \int\left(v_{d c}-v_{d c_{-} r e f}\right) d t .
$$

Meanwhile, the component $V_{s \beta}$ is synthesized to emulate an equivalent reactance $X_{e}$, which is designed as a function of current magnitude.

$$
V_{s \beta}=X_{e} \cdot I_{a b}, X_{e}=f\left(I_{a b}\right) .
$$

Fig. 3 illustrates the entire control block diagram of SSOP. Apart from the control strategies mentioned above, an innerloop voltage control is also utilized to track the SSOP output voltage $v_{s}$ with the reference voltage $v_{s_{-}}$ref. The detailed design of inner-loop voltage control is beyond the scope of this paper but can be found in [18].

On one hand, equation (9) forms a relationship between the SSOP current magnitude $I_{a b}$ and the equivalent impedance $X_{e}$ from the control perspective. On the other hand, the Kirchho- 
ff's voltage law also establishes the relationship between $I_{a b}$ and $X_{e}$ from the physical circuit's perspective. The normal current magnitude $I_{a b_{-} n}$, islanding current magnitude $I_{a b} i$, and short-circuit current magnitude $I_{a b} s$, are respectively given by

$$
\begin{gathered}
I_{a b_{-} n}=\frac{\left|v_{g a}-v_{g b}\right|}{\left|j X_{e}+j \omega L_{\Sigma g}+R_{\Sigma g}\right|}, \\
I_{a b_{-} i}=\frac{\left|v_{g a}\right|}{\left|j X_{e}+R_{l b}+R_{g \Sigma}+j \omega L_{g \Sigma}\right|} \approx \frac{\left|v_{g a}\right|}{\left|j X_{e}+R_{l b}\right|}, \\
I_{a b_{-} s}=\frac{\left|v_{g a}-v_{b}\right|}{\left|j X_{e}+R_{g a}+j \omega L_{g a}\right|} .
\end{gathered}
$$

A simplification has been made in (11) considering that line impedances $R_{g}$ and $\omega L_{g}$ are usually much smaller than the load resistance $R_{l b}$. The combinations of (9) and (10), (9) and (11), (9) and (12) respectively determine the values of $I_{a b \_n}$, $I_{a b_{-}}$, and $I_{a b_{-} s}$, as shown in Fig. 4.

Generally, the function $f$ is designed as a monotonically increasing function such that the SSOP exhibits a stronger block effect when the current magnitude increases. This paper considers the simplest scenario that the equivalent reactance is proportional to the SSOP current magnitude, i.e., $X_{e}=f\left(I_{a b}\right)$ $=k I_{a b}$, where $k$ is the proportional gain.

\section{EXPERIMENTAL RESULTS}

To experimentally verify the proposed technique and its control scheme, a scaled-down hardware prototype has been built in the laboratory. The circuit and control parameters are listed in Table I. The configuration of the hardware setup is displayed in Fig. 5.

Fig. 6 displays the experimental result under the normal operation condition. It is observed that the DC-link voltage $v_{d c}$ can be well regulated around the reference value and the SSOP allows the normal current to flow between the two terminals.

Fig. 7 displays the experimental results under the islanding scenario, where terminal $b$ is disconnected from distribution network at $t_{1}$. Although the loads of terminal $b$ cannot be supplied $v_{g b}$ during the islanding fault, the SSOP is still able to provide an alternative route of power supply and restore the voltage of terminal $b$. As a consequence, $v_{b}$ is hardly affected, as demonstrated in Fig. 7. It can be clearly observed that there is no inrush current or loss of the electricity supply during the islanding fault.

Lastly, Fig. 8 shows the experimental results under the short -circuit fault. The terminal $b$ is grounded through a small impe-dance $Z_{c}$ at $t_{2}$. The emulated short-circuit fault has dropped the voltage magnitude of terminal $b$ to around $60 \mathrm{~V}$, as shown in Fig. 8. Despite the fact, the SSOP immediately synthesizes a back electrical potential to counteract with the voltage sag of $v_{b}$. Through this effort, the SSOP current can be limited within the $0-10 \mathrm{~A}$ normal range. In addition, the shortcircuit fault does not influence the voltage of terminal $a$, as shown in Fig. 8.
TABLE I.

CIRCUIT AND CONTROL PARAMETERS

\begin{tabular}{cc} 
Parameters & Value \\
AC voltage Mag. magnitude & $100 \mathrm{~V}$ \\
Load at terminals $R_{l 1,2}$ & $25 \Omega$ \\
DC-link capacitor $C_{d c}$ & $1880 \mu \mathrm{F}$ \\
Filter capacitor $C_{f 1,2}$ & $15 \mu \mathrm{F}$ \\
Filter inductance $L_{f 1,2}$ & $1 \mathrm{mH}$ \\
Line inductance $L_{g 1,2}$ & $1 \mathrm{mH}$ \\
Line resistance $R_{g 1,2}$ & $1 \Omega$ \\
DC reference $v_{d c_{-} r e f}$ & $125 \mathrm{~V}$ \\
Control gain $k_{p} \quad v_{d c} r e f$ & 0.5 \\
Control gain $k_{i}$ & 2 \\
Switching Fre. $f_{s w}$ & $10 \mathrm{kHz}$ \\
Sampling time $T_{s}$ & $100 \mu \mathrm{s}$ \\
Rated current $I_{m a x}$ & $10 \mathrm{~A}$ \\
Reactance function $f$ & $X_{e}=1.1 I_{a b}$ \\
\hline
\end{tabular}

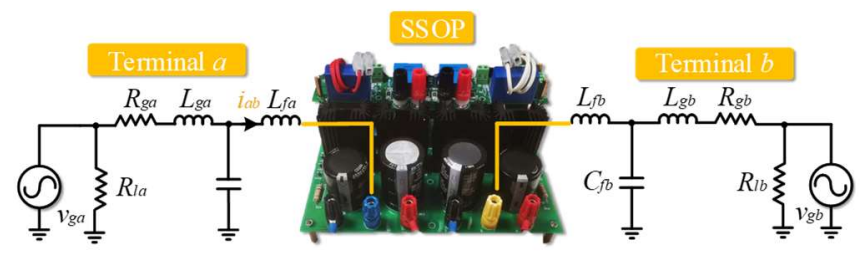

Fig. 5. Circuit configuration of the hardware setup.

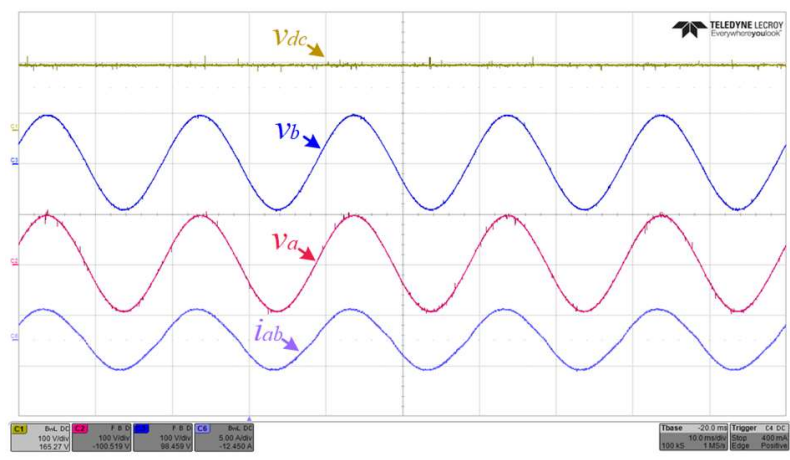

Fig. 6. Experimental waveforms during the normal operation.

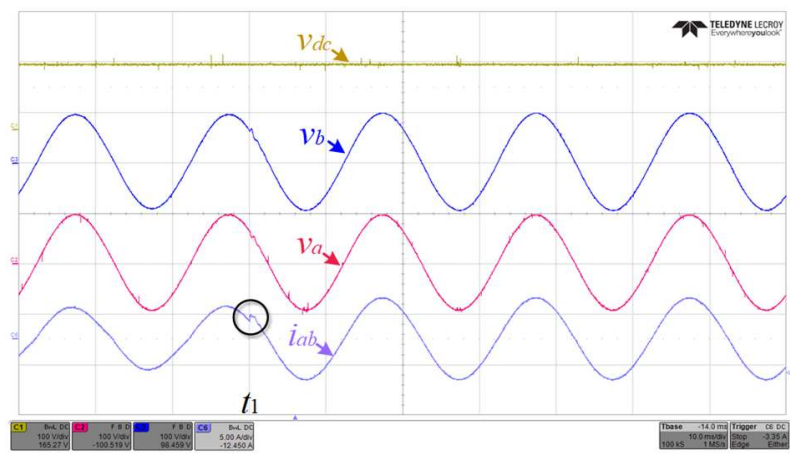

Fig. 7. Experimental results when an islanding fault occurs at terminal $b$. 


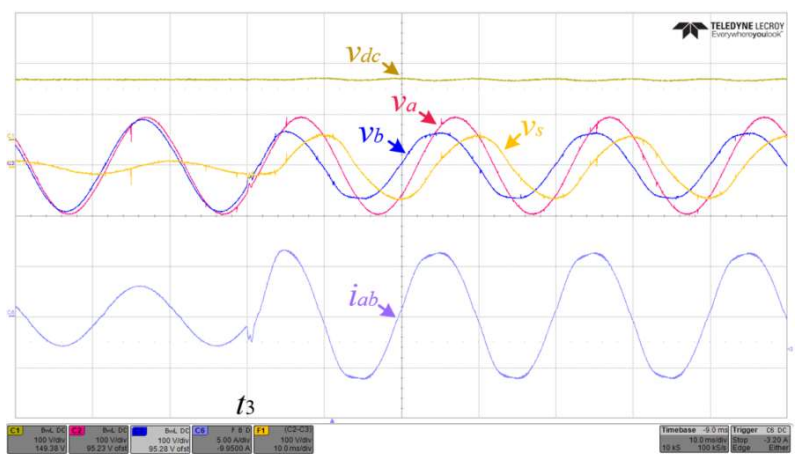

Fig. 8. Experimental waveforms during the short-circuit fault.

\section{CONCLUSIONS}

In this paper, the series soft open point (SSOP) and its control strategy are developed to achieve the fault-tolerant operation of the distribution network. The SSOP is able to autonomously suppress the short-circuit current and restore the supply voltage under an islanding fault scenario. These benefits make the SSOP a promising candidate to improve the reliability of the future power-electronics-based distribution networks. The principle and design guideline of the SSOP are detailed in this paper. Hardware experimental results are provided to validate the feasibility of this proposal.

\section{REFERENCES}

[1] D. E. Olivares et al., "Trends in Microgrid Control," IEEE Trans. Smart Grid, vol. 5, no. 4, pp. 1905-1919, July 2014.

[2] H. Han, X. Hou, J. Yang, J. Wu, M. Su, and J. M. Guerrero, "Review of Power Sharing Control Strategies for Islanding Operation of AC Microgrids," IEEE Trans. Smart Grid, vol. 7, no. 1, pp. 200-215, Jan. 2016.

[3] Y. Qi, Y. Tang, K. R. R. Potti, and K. Rajashekara, "Robust Power Sharing Control for Parallel Three-phase Inverters Against Voltage Measurement Errors," IEEE Trans. Power Electron., vol. 35, no. 12, pp. 13590-13601, Dec. 2020.

[4] Y. Qi, H. Deng, J. Wang, and Y. Tang, "Passivity-Based Synchronization Stability Analysis for Power-Electronic-Interfaced Distributed Generations," IEEE Trans. Sustain. Energy, vol. 12, no. 2, pp. 1141-1150, April 2021.

[5] W. Zhang, D. Xu, P. N. Enjeti, H. Li, J. T. Hawke, and H. S. Krishnamoorthy, "Survey on Fault-Tolerant Techniques for Power
Electronic Converters," IEEE Trans. Power Electron., vol. 29, no. 12, pp. 6319-6331, Dec. 2014.

[6] M. A. Shahab, B. Mozafari, S. Soleymani, N. M. Dehkordi, H. M. Shourkaei, and J. M. Guerrero, "Distributed Consensus-Based Fault Tolerant Control of Islanded Microgrids," IEEE Trans. Smart Grid, vol. 11, no. 1, pp. 37-47, Jan. 2020.

[7] D. Zhou, H. Qiu, S. Yang, and Y. Tang, "Submodule Voltage Similarity-Based Open-Circuit Fault Diagnosis for Modular Multilevel Converters," IEEE Trans. Power Electronics, vol. 34, no. 8, pp. 80088016, Aug. 2019.

[8] X. Lin, Z. Liang, Y. Zheng, Y. Lin, and Y. Kang, "A Current Limiting Strategy With Parallel Virtual Impedance for Three-Phase Three-Leg Inverter Under Asymmetrical Short-Circuit Fault to Improve the Controllable Capability of Fault Currents," IEEE Trans. Power Electronics, vol. 34, no. 8, pp. 8138-8149, Aug. 2019.

[9] J. M. Bloemink, "Distribution-level power electronics: soft open points," London, United Kingdom, 2013.

[10] J. M. Bloemink and T. C. Green, "Increasing distributed generation penetration using soft normally-open points," in Proc. IEEE PES Gen. Meeting, 2010, pp. 1-8.

[11] K. S. Fuad, H. Hafezi, K. Kauhaniemi, and H. Laaksonen, "Soft Open Point in Distribution Networks," IEEE Access, vol. 8, pp. 210550210565, 2020

[12] W. Cao, J. Wu, N. Henkins, C. Wang, and T. Green. "Operating principle of Soft Open Points for electrical distribution network operation," Appl. Energy, vol. 164, pp. 245-257, 2016.

[13] H. Ji, C. Wang, P. Li, F. Ding, and J. Wu, "Robust Operation of Soft Open Points in Active Distribution Networks With High Penetration of Photovoltaic Integration," IEEE Trans. Sustain. Energy, vol. 10, no. 1, pp. 280-289, Jan. 2019.

[14] H. Hafezi and R. Faranda, "Dynamic Voltage Conditioner: A New Concept for Smart Low-Voltage Distribution Systems," IEEE Trans. Power Electron., vol. 33, no. 9, pp. 7582-7590, Sept. 2018

[15] Y. W. Li, D. M. Vilathgamuwa, F. Blaabjerg, and P. C. Loh, "A Robust Control Scheme for Medium-Voltage-Level DVR Implementation," IEEE Trans. Ind. Electron., vol. 54, no. 4, pp. 2249-2261, Aug. 2007.

[16] S. Y. Hui, C. K. Lee, and F. F. Wu, "Electric Springs-A New Smart Grid Technology," IEEE Trans. Smart Grid, vol. 3, no. 3, pp. 15521561, Sept. 2012.

[17] Y. Qi, T. Yang, J. Fang, Y. Tang, K. R. R. Potti, and K. Rajashekara, "Grid Inertia Support Enabled by Smart Loads," IEEE Trans. Power Electron., vol. 36, no. 1, pp. 947-957, Jan. 2021.

[18] Y. Qi, P. Lin, Y. Wang, and Y. Tang, "Two-Dimensional ImpedanceShaping Control With Enhanced Harmonic Power Sharing for InverterBased Microgrids," IEEE Trans. Power Electron., vol. 34, no. 11, pp. 11407-11418, Nov. 2019. 